\title{
On the Existence of Positive Solutions for a Fourth-Order Boundary Value Problem
}

\author{
Yumei Zou \\ Department of Statistics and Finance, Shandong University of Science and Technology, Qingdao 266590, China \\ Correspondence should be addressed to Yumei Zou; sdzouym@126.com
}

Received 10 May 2017; Accepted 17 July 2017; Published 14 August 2017

Academic Editor: Xinguang Zhang

Copyright (C) 2017 Yumei Zou. This is an open access article distributed under the Creative Commons Attribution License, which permits unrestricted use, distribution, and reproduction in any medium, provided the original work is properly cited.

By using the method of order reduction and the fixed point index, the existence of positive solutions for a fourth-order boundary value problem is studied. We provide conditions under which the existence results hold. Such conditions are related to the first eigenvalue corresponding to the relevant linear differential equation with dependence on the derivatives of unknown function.

\section{Introduction}

In this paper, we consider the following fourth-order ordinary differential equation boundary value problem (BVP):

$$
\begin{aligned}
& u^{(4)}(t)=f\left(t, u(t), u^{\prime}(t), u^{\prime \prime}(t), u^{\prime \prime \prime}(t)\right), \\
& t \in[0,1], \\
& u(0)=u^{\prime}(0)=u^{\prime \prime}(1)=u^{\prime \prime \prime}(1)=0,
\end{aligned}
$$

where $f:[0,1] \times \mathbb{R}_{+}^{3} \times \mathbb{R}_{-} \rightarrow \mathbb{R}_{+}$is continuous.

BVP (1) is used to model such phenomena as the deformations of an elastic beam in equilibrium state, whose one endpoint is fixed and the other is freed. In mechanics, BVP (1) is called cantilever beam equation. Owing to its importance in mechanics, the existence of solutions to this problem has been studied by many authors; see [1-7] and references therein.

Very recently, Li [3] used the theory of the fixed point index to discuss the existence of solutions of BVP (1) when $f\left(t, x_{0}, x_{1}, x_{2}, x_{3}\right)$ is superlinear or sublinear growth on $x_{0}, x_{1}, x_{2}, x_{3}$. It should be remarked that two constants 1 and $\lambda_{1}$, where $\lambda_{1}$ is the first eigenvalue of the linear eigenvalue problem corresponding to BVP (1), play an important role in the discussion. The first eigenvalue principles were also used in [8-24].

However, none of these known results use the first eigenvalues of the corresponding linear differential equation that contains the derivative of the unknown function. This is because the presence of derivatives in linear eigenvalue problem will make the study extremely difficult. To overcome this difficulty, we employ the method of order reduction to develop spectral properties of associated linear differential equation that contains the derivative of the unknown function. Then, we use the fixed point index theory to investigate the existence results of positive solutions for BVP (1) under some conditions concerning the first eigenvalue corresponding to the relevant linear eigenvalue problem that contains the derivative of the unknown function. It should be noted that the method of order reduction was also used in $[7,25]$ to transform higher order boundary value problem to a lower order integrodifferential equation.

\section{Main Results}

Let $\mathbb{R}_{+}=[0,+\infty)$ and $\mathbb{R}_{-}=(-\infty, 0]$. We use the classical Banach space $C[0,1], C^{1}[0,1]$ with the norm $\|u\|_{C}=$ $\max _{t \in[0,1]}|u(t)|,\|u\|_{C^{1}}=\max \left\{\|u\|_{C},\left\|u^{\prime}\right\|_{C}\right\}$, respectively. Let $P$ denote the cone of all nonnegative functions in $C[0,1]$.

We assume the following hypothesis holds throughout this paper.

$$
\left(H_{1}\right) f \in C\left([0,1] \times \mathbb{R}_{+}^{3} \times \mathbb{R}_{-}, \mathbb{R}_{+}\right)
$$

Firstly, we consider the existence of solutions to BVP (1) when the nonlinearity $f$ satisfies the sublinear growth 
property. To this end, we use the method of order reduction to transform BVP (1). For $v \in C[0,1]$, we set

$$
\begin{aligned}
& \left(T_{3} v\right)(t)=\int_{t}^{1} v(s) d s \\
& \left(T_{2} v\right)(t)=\int_{0}^{1} G_{2}(t, s) v(s) d s \\
& \left(T_{1} v\right)(t)=\int_{0}^{1} G_{1}(t, s) v(s) d s
\end{aligned}
$$

where

$$
\begin{aligned}
& G_{2}(t, s)= \begin{cases}t, & 0 \leq t \leq s \leq 1, \\
s, & 0 \leq s \leq t \leq 1,\end{cases} \\
& G_{1}(t, s)= \begin{cases}\frac{t^{2}}{2}, & 0 \leq t \leq s \leq 1, \\
\frac{2 t s-s^{2}}{2}, & 0 \leq s \leq t \leq 1 .\end{cases}
\end{aligned}
$$

Then we have $\left(T_{1} v\right)^{\prime \prime \prime}=\left(T_{2} v\right)^{\prime \prime}=\left(T_{3} v\right)^{\prime}=-v$. From the above expressions, we easily see that $T_{i}: C[0,1] \rightarrow C[0,1]$ $(i=1,2,3)$ are three completely continuous linear operators, and $G_{1}$ and $G_{2}$ have the following properties:

$$
\begin{aligned}
\frac{t^{2}}{2} s & \leq G_{1}(t, s) \leq s, \\
t s & \leq G_{2}(t, s) \leq s,
\end{aligned}
$$

$$
\forall t, s \in[0,1] .
$$

Hence we conclude that

$$
\begin{aligned}
T_{1}\left(P_{1}\right) & \subset P_{1} \\
& =\left\{u \in C[0,1]: u(t) \geq \frac{t^{2}}{2}\|u\|_{C}, t \in[0,1]\right\} .
\end{aligned}
$$

Using the above transformations $T_{i}(i=1,2,3)$, BVP (1) can be converted into the equivalent nonlinear integral equation:

$$
\begin{aligned}
& v(t) \\
& \quad=\int_{t}^{1} f\left(s,\left(T_{1} v\right)(s),\left(T_{2} v\right)(s),\left(T_{3} v\right)(s),-v(s)\right) d s,
\end{aligned}
$$

$$
v \in C[0,1] .
$$

Define an operator $A$ by

$$
\begin{array}{r}
(A v)(t) \\
=\int_{t}^{1} f\left(s,\left(T_{1} v\right)(s),\left(T_{2} v\right)(s),\left(T_{3} v\right)(s),-v(s)\right) d s, \\
v \in C[0,1] .
\end{array}
$$

Then the existence of a positive solution of BVP (1) is equivalent to the existence of a nontrivial fixed point of $A$ on
$P$. Now condition $\left(H_{1}\right)$ implies that $A: P \rightarrow P$ is a completely continuous operator.

For $\mathbf{a}=\left(a_{1}, b_{1}, c_{1}, d_{1}\right) \in \mathbb{R}_{+}^{4}$ with $a_{1}^{2}+b_{1}^{2} \neq 0$, we define linear operator $K_{\mathbf{a}}$ by

$$
\begin{aligned}
& \left(K_{\mathbf{a}} v\right)(t)=\int_{t}^{1}\left(a_{1}\left(T_{1} v\right)(s)+b_{1}\left(T_{2} v\right)+c_{1}\left(T_{3} v\right)(s)\right. \\
& \left.+d_{1} v(s)\right) d s, \quad v \in C[0,1] .
\end{aligned}
$$

Clearly, $K_{\mathrm{a}}: P \rightarrow P$ is a completely continuous linear operator.

Lemma 1. For the operator $K_{\mathrm{a}}$ defined by (8), the spectral radius $r\left(K_{\mathbf{a}}\right) \neq 0$ and $K_{\mathbf{a}}$ had unique positive unit eigenfunction $\varphi_{\mathbf{a}}$ corresponding to its first eigenvalue $\lambda_{1}=\left(r\left(K_{\mathbf{a}}\right)\right)^{-1}$.

Proof. Take $v(t)=1-t$; clearly $v \in P$; by (3) we have

$$
\begin{aligned}
\left(T_{1} v\right)(t) & \geq \int_{0}^{t} \frac{2 t s-s^{2}}{2}(1-s) d s \geq \int_{0}^{t} \frac{t s}{2}(1-s) d s \\
& =\frac{t^{3}}{12}(3-2 t) \geq \frac{t^{3}}{12} \\
\left(T_{2} v\right)(t) & \geq \int_{0}^{t} s(1-s) d s=\frac{t^{2}}{6}(3-2 t) \geq \frac{t^{2}}{6}
\end{aligned}
$$

So we have

$$
\begin{aligned}
\left(K_{\mathbf{a}} v\right)(t) & \geq a_{1} \int_{t}^{1}\left(T_{1} v_{0}\right)(s) d s+b_{1} \int_{t}^{1}\left(T_{2} v_{0}\right)(s) d s \\
& \geq\left(\frac{a_{1}}{48}+\frac{b_{1}}{18}\right) v(t) .
\end{aligned}
$$

Thus there exists a constant $c>0$ such that $c\left(K_{\mathrm{a}} v\right)(t) \geq$ $v(t), t \in[0,1]$. From Lemma 2 in [22], we know that the spectral radius $r\left(K_{\mathbf{a}}\right) \neq 0$ and $K_{\mathbf{a}}$ had a positive eigenfunction corresponding to its first eigenvalue $\lambda_{1}=\left(r\left(K_{\mathbf{a}}\right)\right)^{-1}$. Hence Lemma 1 holds.

It follows from the definition of operator $K_{\mathrm{a}}$ that the function $\varphi_{\mathbf{a}}$, corresponding to the first eigenvalue $\lambda_{1}=\left(r\left(K_{\mathbf{a}}\right)\right)^{-1}$ of the operator $K_{\mathbf{a}}$, belongs to $C^{4}[0,1]$ and satisfies the equation

$$
\begin{aligned}
& \left(T_{1} \varphi_{\mathbf{a}}\right)^{(4)}(t)=\lambda_{1}\left(a_{1}\left(T_{1} \varphi_{\mathbf{a}}\right)+b_{1}\left(T_{1} \varphi_{\mathbf{a}}\right)^{\prime}+c_{1}\left(T_{1} \varphi_{\mathbf{a}}\right)^{\prime \prime}\right. \\
& \left.\quad-d_{1}\left(T_{1} \varphi_{\mathbf{a}}\right)^{\prime \prime \prime}\right), \quad t \in[0,1] \\
& \left(T_{1} \varphi_{\mathbf{a}}\right)(0)=\left(T_{1} \varphi_{\mathbf{a}}\right)^{\prime}(0)=\left(T_{1} \varphi_{\mathbf{a}}\right)^{\prime \prime}(1)=\left(T_{1} \varphi_{\mathbf{a}}\right)^{\prime \prime \prime}(1) \\
& \quad=0
\end{aligned}
$$

In particular, for $\mathbf{a}=\left(a_{1}, b_{1}, 0,0\right) \in \mathbb{R}_{+}^{4}$, the positive eigenfunction $\varphi_{\mathbf{a}}$ of the operator $K_{\mathbf{a}}$ corresponding to the first 
eigenvalue $\lambda_{1}=\left(r\left(K_{\mathbf{a}}\right)\right)^{-1}$ belongs to $C^{4}[0,1]$ and satisfies the equation

$$
\begin{aligned}
\left(T_{1} \varphi_{\mathbf{a}}\right)^{(4)}(t) & =\lambda_{1}\left(a_{1}\left(T_{1} \varphi_{\mathbf{a}}\right)+b_{1}\left(T_{1} \varphi_{\mathbf{a}}\right)^{\prime}\right), \\
\left(T_{1} \varphi_{\mathbf{a}}\right)(0) & =\left(T_{1} \varphi_{\mathbf{a}}\right)^{\prime}(0)=\left(T_{1} \varphi_{\mathbf{a}}\right)^{\prime \prime}(1) \\
& =\left(T_{1} \varphi_{\mathbf{a}}\right)^{\prime \prime \prime}(1)=0 .
\end{aligned}
$$

Theorem 2. If $f$ satisfies $\left(H_{1}\right)$ and the following conditions, then BVP (1) has at least one positive solution.

$\left(H_{2}\right)$ There are $\mathbf{a}=\left(a_{1}, b_{1}, c_{1}, d_{1}\right) \in \mathbb{R}_{+}^{4}$ with $a_{1}^{2}+b_{1}^{2} \neq 0$ and $c>0$ such that $r\left(K_{\mathbf{a}}\right)<1$ and

$$
\begin{array}{r}
f\left(t, x_{1}, x_{2}, x_{3}, x_{4}\right) \leq a_{1} x_{1}+b_{1} x_{2}+c_{1} x_{3}+d_{1}\left|x_{4}\right|+c, \\
\left(x_{1}, x_{2}, x_{3}, x_{4}\right) \in \mathbb{R}_{+}^{3} \times \mathbb{R}_{-} .
\end{array}
$$

$\left(H_{3}\right)$ There are $\mathbf{b}=\left(a_{2}, b_{2}, c_{2}, d_{2}\right) \in \mathbb{R}_{+}^{4}$ with $a_{2}^{2}+b_{2}^{2} \neq 0$ and $r>0$ such that $r\left(K_{\mathbf{b}}\right)>1$ and

$$
\begin{aligned}
f\left(t, x_{1}, x_{2}, x_{3}, x_{4}\right) \geq & a_{1} x_{1}+b_{1} x_{2}+c_{1} x_{3}+d_{1}\left|x_{4}\right|, \\
& \left(x_{1}, x_{2}, x_{3}, x_{4}\right) \in[0, r]^{3} \times[-r, 0] .
\end{aligned}
$$

Proof. Let $W_{1}=\{v \in P: v=\lambda A v$, for some $\lambda \in[0,1]\}$. We will prove that $W_{1}$ is a bounded set. In fact, if $v \in W_{1}$, by the definition of $W_{1}, v=\lambda A v$ for some $\lambda \in[0,1]$. Hence we have

$$
\begin{array}{r}
v(t) \leq(A v)(t) \\
=\int_{t}^{1} f\left(s,\left(T_{1} v\right)(s),\left(T_{2} v\right)(s),\left(T_{3} v\right)(s),-v(s)\right) d s, \\
v v \in P, t \in[0,1] .
\end{array}
$$

By $\left(H_{2}\right)$, we have $v(t) \leq\left(K_{\mathrm{a}} v\right)(t)+c(1-t)$, which can be rewritten in the form $\left(I-K_{\mathrm{a}}\right) v \leq \widetilde{v}_{0}$, where $\widetilde{v}_{0}(t)=c(1-t)$. The condition $r\left(K_{\mathrm{a}}\right)<1$ ensures that $I-K_{\mathrm{a}}$ has a bounded inverse operator $\left(I-K_{\mathbf{a}}\right)^{-1}$ which is given by $\left(I-K_{\mathbf{a}}\right)^{-1}=$ $I+K_{\mathrm{a}}+K_{\mathrm{a}}^{2}+\cdots+K_{\mathrm{a}}+\cdots$. It follows from $K_{\mathrm{a}}(P) \subset P$ that $\left(I-K_{\mathbf{a}}\right)^{-1}(P) \subset P$. So we have $v \leq\left(I-K_{\mathbf{a}}\right)^{-1} \widetilde{v}_{0}$ and $W_{1}$ is bounded. Select $R>\max \left\{r, \sup \left\{\|v\|_{C}: v \in W_{1}\right\}\right\}$; we have $v \neq \lambda A v, v \in \partial \Omega_{R}, \lambda \in[0,1]$, where $\Omega_{R}=\{u \in C[0,1]$ : $\left.\|u\|_{C}<R\right\}$. Now Lemma 2.3.1 in [26] implies

$$
i\left(A, \Omega_{R} \cap P, P\right)=1 .
$$

Let $\varphi_{\mathbf{b}}$ be the positive eigenvalue function of $K_{\mathbf{b}}$ (with replacement of $\mathbf{a}$ by $\mathbf{b}$ in Lemma 1). We may suppose that $A$ has no fixed point on $\partial \Omega_{r} \cap P$ (otherwise, the proof is finished). Now we shall show that

$$
v-A v \neq \lambda \varphi_{\mathrm{b}}, \quad \forall v \in \partial \Omega_{r} \cap P, \lambda \geq 0,
$$

where $\Omega_{r}=\left\{u \in C[0,1]:\|u\|_{C}<r\right\}$. In fact, if (17) is not valid, there exist $v_{0} \in \partial \Omega_{r} \cap P$ and $\lambda_{0} \geq 0$ such that
$v_{0}-A v_{0}=\lambda_{0} \varphi_{\mathbf{b}}$. Hence we have $\lambda_{0}>0$. Since $v_{0} \in \partial \Omega_{r} \cap P$, by the definitions of $P$ and $T_{i}(i=1,2,3)$, we have

$$
0 \leq v_{1}(t),\left(T_{1} v\right)(t),\left(T_{2} v\right)(t),-\left(T_{3} v\right)(t) \leq r,
$$$$
t \in[0,1] \text {. }
$$

Hence from $\left(\mathrm{H}_{3}\right)$, we obtain that

$$
\begin{aligned}
f( & \left.t,\left(T_{1} v_{0}\right)(t),\left(T_{2} v_{0}\right)(t),\left(T_{3} v_{0}\right)(t),-v_{0}(t)\right) \\
\geq & a_{2}\left(T_{1} v_{0}\right)(t)+b_{2}\left(T_{2} v_{0}\right)(t)+c_{2}\left(T_{3} v_{0}\right)(t) \\
& +d_{2} v_{0}(t)
\end{aligned}
$$

from which it follows that

$$
v_{0}=A v_{0}+\lambda_{0} \varphi_{\mathbf{b}} \geq K_{\mathbf{b}} v_{0}+\lambda_{0} \varphi_{\mathbf{b}} .
$$

Set $\lambda^{*}=\sup \left\{\lambda: v_{0} \geq \lambda \varphi_{\mathbf{b}}\right\}$. It is easy to see that $\lambda^{*}>0$ and $v_{0} \geq \lambda^{*} \varphi_{\mathbf{b}}$. It follows from $K_{\mathbf{b}}(P) \subset P$ that $K_{\mathbf{b}} v_{0} \geq \lambda^{*} K_{\mathbf{b}} \varphi_{\mathbf{b}}=$ $\lambda^{*} r\left(K_{\mathbf{b}}\right) \varphi_{\mathbf{b}}$. Therefore, by (20),

$$
v_{0}=A v_{0}+\lambda_{0} \varphi_{\mathbf{b}} \geq r\left(K_{\mathbf{b}}\right) \lambda^{*} \varphi_{\mathbf{b}}+\lambda_{0} \varphi_{\mathbf{b}}
$$

which contradicts the definition of $\lambda^{*}$. Hence (17) is true and we have from Corollary 2.3.1 in [26] that

$$
i\left(A, \Omega_{r} \cap P, P\right)=0 .
$$

By (16) and (22), we have that

$$
\begin{aligned}
i( & \left.A,\left(\Omega_{R} \cap P\right) \backslash\left(\overline{\Omega_{r}} \cap P\right), P\right) \\
\quad & i\left(A, \Omega_{R} \cap P, P\right)-i\left(A, \Omega_{r} \cap P, P\right)=1 .
\end{aligned}
$$

Then $A$ has at least one fixed point in $\left(\Omega_{R} \cap P\right) \backslash\left(\overline{\Omega_{r}} \cap P\right)$ which means that BVP (1) has a positive solution. The proof of Theorem 2 is completed.

Next, we consider the existence of solutions to BVP (1) when the nonlinearity $f$ satisfies the superlinear growth condition. In this case, we assume that $f\left(t, x_{0}, x_{1}, x_{2}, x_{3}\right)$ is independent of $x_{2}$ and $x_{3}$; that is, we consider the following simplified BVP:

$$
\begin{aligned}
u^{(4)}(t) & =f\left(t, u(t), u^{\prime}(t)\right) \\
u(0) & =u^{\prime}(0)=u^{\prime \prime}(1)=u^{\prime \prime \prime}(1)=0 .
\end{aligned}
$$

As in [3], a positive solution of BVP (24) is equivalent to a nontrivial fixed point of $B$ defined by

$$
(B u)(t)=\int_{0}^{1} G(t, s) f\left(s, u(s), u^{\prime}(s)\right) d s,
$$

$$
u \in C^{1}[0,1],
$$

where $G(t, s)$ is Green's function of the homogeneous linear problem $u^{(4)}(t)=0, u(0)=u^{\prime}(0)=u^{\prime \prime}(1)=u^{\prime \prime \prime}(1)=0$, which is explicitly given by

$$
G(t, s)= \begin{cases}\frac{1}{6} t^{2}(3 s-t), & 0 \leq t \leq s \leq 1, \\ \frac{1}{6} s^{2}(3 t-s), & 0 \leq s \leq t \leq 1 .\end{cases}
$$


From (26) we can easily verify that $G(t, s)$ has the following properties:

$$
\frac{1}{3} t^{2} s^{2} \leq G(t, s) \leq \frac{1}{2} t^{2}\left(\text { or } \frac{1}{2} s^{2}\right), \quad t, s \in[0,1] .
$$

For $\mathbf{c}=\left(a_{1}, b_{1}\right) \in \mathbb{R}_{+}^{2}$ with $a_{1}^{2}+b_{1}^{2} \neq 0$ (we also use $\mathbf{c}$ denoting $\left.\left(a_{1}, b_{1}, 0,0\right)\right)$. Define a linear integral operator $L$ by

$$
\begin{aligned}
\left(L_{\mathrm{c}} u\right)(t)=\int_{0}^{1} G(t, s)\left(a_{1} u(s)+b_{1} u^{\prime}(s)\right) d s, & \\
u & \in C^{1}[0,1] .
\end{aligned}
$$

In what follows we work on the Banach space $C^{1}[0,1]$ and utilize the cone

$$
\begin{aligned}
P_{3} & =\left\{u \in C^{1}[0,1]: u(0)=0, u(t)\right. \\
& \left.\geq \frac{1}{2} t^{2}\|u\|_{C}, u^{\prime}(t) \geq t\left\|u^{\prime}\right\|_{C}, 0 \leq t \leq 1\right\} .
\end{aligned}
$$

Lemma 3 (see $[3,6]$ ). If $u \in P_{3}$, then $\left\|u^{\prime}\right\|_{C^{1}}=\|u\|_{C}$ and $(1 / 2)\|u\|_{C^{1}} \leq\|u\|_{C} \leq\|u\|_{C^{1}}$.

Lemma 4 (see $[3,6]$ ). $B, L: P_{3} \rightarrow P_{3}$ are two completely continuous operators.

Based on the definition of operator $L_{\mathfrak{c}}$, (12) can be rewritten in the following form:

$$
T_{1} \varphi_{\mathbf{c}}=\left(r\left(K_{\mathbf{c}}\right)\right)^{-1} L_{\mathbf{c}}\left(T_{1} \varphi_{\mathbf{c}}\right)
$$

which means that the operator $L_{\mathbf{c}}$ has an eigenvalue $\left(r\left(K_{\mathrm{c}}\right)\right)^{-1}$, and its related eigenfunction can be taken by $T_{1} \varphi_{c}$. In fact, we have $r\left(K_{\mathfrak{c}}\right)=r\left(L_{\mathbf{c}}\right)$ from equivalent differential equation (12).

Theorem 5. If $f$ satisfies $\left(H_{1}\right)$ and the following conditions, then BVP (24) has at least one positive solution.

$\left(H_{4}\right)$ There are $\mathbf{c}=\left(a_{1}, b_{1}\right) \in \mathbb{R}_{+}^{2}$ with $a_{1}^{2}+b_{1}^{2} \neq 0$ and $c>0$ such that $r\left(L_{\mathbf{c}}\right)>1$ and

$$
\begin{aligned}
f\left(t, x_{1}, x_{2}\right) \geq a_{1} x_{1}+b_{1} x_{2}-c, & \\
& \left(x_{1}, x_{2}\right) \in \mathbb{R}^{2}, t \in[0,1] .
\end{aligned}
$$

$\left(H_{5}\right)$ There are $\mathbf{d}=\left(a_{2}, b_{2}\right) \in \mathbb{R}_{+}^{2}$ with $a_{2}^{2}+b_{2}^{2} \neq 0$ and $\delta>0$ such that $r\left(L_{\mathbf{d}}\right)<1$ and

$$
f\left(t, x_{1}, x_{2}\right) \leq a_{2} x_{1}+b_{2} x_{2},
$$

$$
\left(x_{1}, x_{2}\right) \in[0, \delta]^{2}, t \in[0,1] .
$$

Proof. Set $\varepsilon>0$ such that $(1-\varepsilon) r\left(L_{\mathbf{c}}\right)>1$. Take $R>\max \{\delta$, $\left.30 c / \varepsilon\left(2 a_{1}+5 b_{1}\right)\right\}$. For every $u \in \partial \Omega_{R} \cap P_{3}$, it follows from (27) and Lemma 3 that

$$
\begin{aligned}
B u \geq & L_{\mathbf{c}} u-c \int_{0}^{1} G(t, s) d s \\
\geq & (1-\varepsilon) L_{\mathbf{c}} u+\varepsilon L_{\mathfrak{c}} u-\frac{c}{2} t^{2} \\
\geq & (1-\varepsilon) L_{\mathfrak{c}} u \\
& \quad+\varepsilon \int_{0}^{1} \frac{1}{3} t^{2} s^{2}\left(\frac{a_{1}}{2} s^{2}\|u\|_{C^{1}}+b_{1} s\|u\|_{C^{1}}\right) d s-\frac{c}{2} t^{2} \\
\geq & (1-\varepsilon) L_{\mathbf{c}} u+t^{2}\left(\varepsilon\|u\|_{C^{1}}\left(\frac{a_{1}}{30}+\frac{b_{1}}{12}\right)-\frac{c}{2}\right) \\
\geq & (1-\varepsilon) L_{\mathbf{c}} u .
\end{aligned}
$$

We may suppose that $B$ has no fixed point on $\partial \Omega_{R} \cap P_{3}$ (otherwise, the proof is finished). Now we show that

$$
u-B u \neq \mu T_{1} \varphi_{c}, \quad \forall u \in \partial \Omega_{R} \cap P_{3}, \quad \mu \geq 0,
$$

where $\varphi_{\mathrm{c}}$ is the positive eigenfunction of operator $S_{\mathrm{c}}$ related to its first eigenvalue $\left(r\left(S_{\mathbf{c}}\right)\right)^{-1}$, and $T_{1} \varphi_{\mathbf{c}} \in P_{3} \backslash\{\theta\}$ follows from (5).

In fact, if (34) is not valid, there exist $u_{0} \in \partial \Omega_{R} \cap P_{3}$ and $\mu_{0} \geq 0$ such that

$$
u_{0}-B u_{0}=\mu_{0} T_{1} \varphi_{c} .
$$

Hence we have $\mu_{0}>0$. Set $\mu^{*}=\sup \left\{\mu: u_{0} \geq \mu T_{1} \varphi_{c}\right\}$. It is easy to see that $\mu^{*}>0$ and $u_{0} \geq \mu^{*} T_{1} \varphi_{\mathrm{c}}$. Since $u_{0} \in \partial \Omega_{R} \cap P_{3}$, by (33), we obtain that

$$
u_{0}=B u_{0}+\mu_{0} T_{1} \varphi_{c} \geq(1-\varepsilon) L_{c} u_{0}+\mu_{0} T_{1} \varphi_{c} .
$$

Therefore by (30), $u_{0} \geq \mu^{*}(1-\varepsilon) r\left(L_{c}\right) T_{1} \varphi_{c}>\mu^{*} T_{1} \varphi_{c}$, which contradicts the definition of $\mu^{*}$. Hence (34) is true and we have from Corollary 2.3.1 in [26] that

$$
i\left(A, \Omega_{R} \cap P_{3}, P_{3}\right)=0 .
$$

Next, we prove that

$$
u \neq \mu B u, \quad u \in \partial \Omega_{\delta} \cap P_{3}, \mu \in[0,1] .
$$

If this is false, then there exist $u_{1} \in \partial \Omega_{\delta} \cap P_{3}$ and $\mu_{1} \in[0,1]$ such that $u_{1}=\mu_{1} B u_{1}$. It follows from Lemma 3 that $0 \leq$ $u_{1}(t), u_{1}^{\prime}(t) \leq \delta, t \in[0,1]$. Then by assumption $\left(H_{5}\right)$, we have $u_{1}=\mu_{1} B u_{1} \leq \mu_{1} L_{\mathbf{d}} u_{1} \leq L_{\mathbf{d}} u_{1}$. Notice that $r\left(L_{\mathbf{d}}\right)<1$; we obtain $u_{1} \leq\left(I-L_{\mathbf{d}}\right)^{-1} \theta=\theta$, which is a contradiction with $u_{1} \in \partial \Omega_{\delta} \cap P_{3}$. Hence, (38) holds. Then by Lemma 2.3.1 in [26], we have

$$
i\left(A, \Omega_{\delta} \cap P_{3}, P_{3}\right)=1 .
$$

By (37) and (39), we have that

$$
\begin{aligned}
& i\left(B,\left(\Omega_{R} \cap P_{3}\right) \backslash\left(\overline{\Omega_{\delta}} \cap P_{3}\right), P_{3}\right) \\
& \quad=i\left(B, \Omega_{R} \cap P_{3}, P_{3}\right)-i\left(B, \Omega_{\delta} \cap P_{3}, P_{3}\right)=-1 .
\end{aligned}
$$

Then $B$ has at least one fixed point in $\left(\Omega_{R} \cap P_{3}\right) \backslash\left(\overline{\Omega_{\delta}} \cap P_{3}\right)$ which means that BVP (24) has a positive solution. The proof of Theorem 5 is completed. 


\section{Conflicts of Interest}

The author declares that they have no conflicts of interest.

\section{Acknowledgments}

The project is supported by NNSF of China (11371221; 11571207)

\section{References}

[1] R. P. Agarwal and D. O'Regan, "Multiplicity results for singular conjugate, focal and (n,p) problems," Journal of Differential Equations, vol. 170, no. 1, pp. 142-156, 2001.

[2] R. P. Agarwal and D. O’Regan, “Twin solutions to singular boundary value problems," Proceedings of the American Mathematical Society, vol. 128, no. 7, pp. 2085-2094, 2000.

[3] Y. Li, "Existence of positive solutions for the cantilever beam equations with fully nonlinear terms," Nonlinear Analysis. Real World Applications, vol. 27, pp. 221-237, 2016.

[4] W. Fan, X. Hao, L. Liu, and Y. Wu, "Nontrivial solutions of singular fourth-order Sturm-Liouville boundary value problems with a sign-changing nonlinear term," Applied Mathematics and Computation, vol. 217, no. 15, pp. 6700-6708, 2011.

[5] F. Minhos, T. Gyulov, and A. I. Santos, "Lower and upper solutions for a fully nonlinear beam equation," Nonlinear Analysis. Theory, Methods \& Applications, vol. 71, no. 1-2, pp. 281-292, 2009.

[6] Q. Yao, "Local existence of multiple positive solutions to a singular cantilever beam equation," Journal of Mathematical Analysis and Applications, vol. 363, no. 1, pp. 138-154, 2010.

[7] Z. Yang and J. Sun, "Positive solutions of a fourth-order boundary value problem involving derivatives of all orders," Communications on Pure and Applied Analysis, vol. 11, no. 5, pp. 16151628, 2012.

[8] Z. Bai, "On positive solutions of a nonlocal fractional boundary value problem," Nonlinear Analysis. Theory, Methods \& Applications, vol. 72, no. 2, pp. 916-924, 2010.

[9] Y. Cui, "Uniqueness of solution for boundary value problems for fractional differential equations," Applied Mathematics Letters, vol. 51, pp. 48-54, 2016.

[10] Y. Cui and Y. Zou, "Existence of solutions for second-order integral boundary value problems," Lithuanian Association of Nonlinear Analysts (LANA). Nonlinear Analysis. Modelling and Control, vol. 21, no. 6, pp. 828-838, 2016.

[11] Y. Cui and J. Sun, “A generalization of Mahadevan's version of the Krein-Rutman theorem and applications to p-Laplacian boundary value problems," Abstract and Applied Analysis, vol. 2012, Article ID 305279, 14 pages, 2012.

[12] Y. Cui and J. Sun, "Existence of multiple positive solutions for fourth-order boundary value problems in Banach spaces," Boundary Value Problems, vol. 107, 2012.

[13] L. Erbe, "Eigenvalue criteria for existence of positive solutions to nonlinear boundary value problems," Mathematical and Computer Modelling, vol. 32, no. 5-6, pp. 529-539, 2000.

[14] F. Sun, L. Liu, X. Zhang, and Y. Wu, "Spectral analysis for a singular differential system with integral boundary conditions," Mediterranean Journal of Mathematics, vol. 13, no. 6, pp. 47634782, 2016.
[15] J. Sun and Y. Cui, "Fixed point theorems for a class of nonlinear operators in Riesz spaces," Fixed Point Theory, vol. 14, no. 1, pp. 185-192, 2013.

[16] K. Q. Lan and W. Lin, "Positive solutions of systems of singular Hammerstein integral equations with applications to semilinear elliptic equations in annuli," Nonlinear Analysis. Theory, Methods \& Applications, vol. 74, no. 18, pp. 7184-7197, 2011.

[17] H. Li and J. Sun, "Positive solutions of superlinear semipositone nonlinear boundary value problems," Computers \& Mathematics with Applications, vol. 61, no. 9, pp. 2806-2815, 2011.

[18] Z. Liu and F. Li, "Multiple positive solutions of nonlinear two-point boundary value problems," Journal of Mathematical Analysis and Applications, vol. 203, no. 3, pp. 610-625, 1996.

[19] L. Liu, F. Sun, X. Zhang, and Y. Wu, "Bifurcation analysis for a singular differential system with two parameters via to topological degree theory," Lithuanian Association of Nonlinear Analysts (LANA). Nonlinear Analysis. Modelling and Control, vol. 22, no. 1, pp. 31-50, 2017.

[20] Y. Wang, L. Liu, and Y. Wu, "Positive solutions for a nonlocal fractional differential equation," Nonlinear Analysis. Theory, Methods \& Applications, vol. 74, no. 11, pp. 3599-3605, 2011.

[21] Z. Yang, "Positive solutions of a 2 nth-order boundary value problem involving all derivatives via the order reduction method," Computers \& Mathematics with Applications, vol. 61, no. 4, pp. 822-831, 2011.

[22] G. Zhang and J. Sun, "Positive solutions of m-point boundary value problems," Journal of Mathematical Analysis and Applications, vol. 291, no. 2, pp. 406-418, 2004.

[23] X. Zhang, L. Liu, Y. Wu, and B. Wiwatanapataphee, “The spectral analysis for a singular fractional differential equation with a signed measure," Applied Mathematics and Computation, vol. 257, pp. 252-263, 2015.

[24] X. Zhang, L. Liu, and Y. Wu, "The eigenvalue problem for a singular higher order fractional differential equation involving fractional derivatives," Applied Mathematics and Computation, vol. 218, no. 17, pp. 8526-8536, 2012.

[25] X. Zhang and L. Liu, "Nontrivial solutions for higher order multi-point boundary value problems," Computers \& Mathematics with Applications, vol. 56, no. 4, pp. 861-873, 2008.

[26] D. J. Guo and V. Lakshmikantham, Nonlinear Problems in Abstract Cones, vol. 5 of Notes and Reports in Mathematics in Science and Engineering, Academic Press, New York, NY, USA, 1988. 


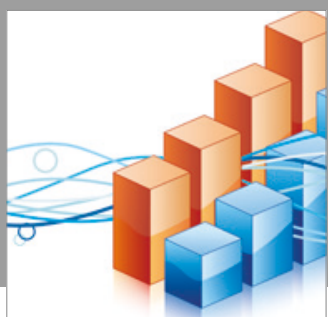

Advances in

Operations Research

vatersals

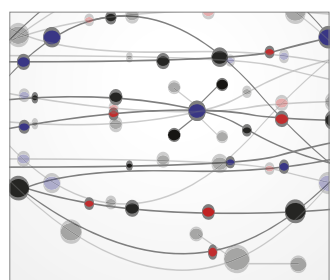

\section{The Scientific} World Journal
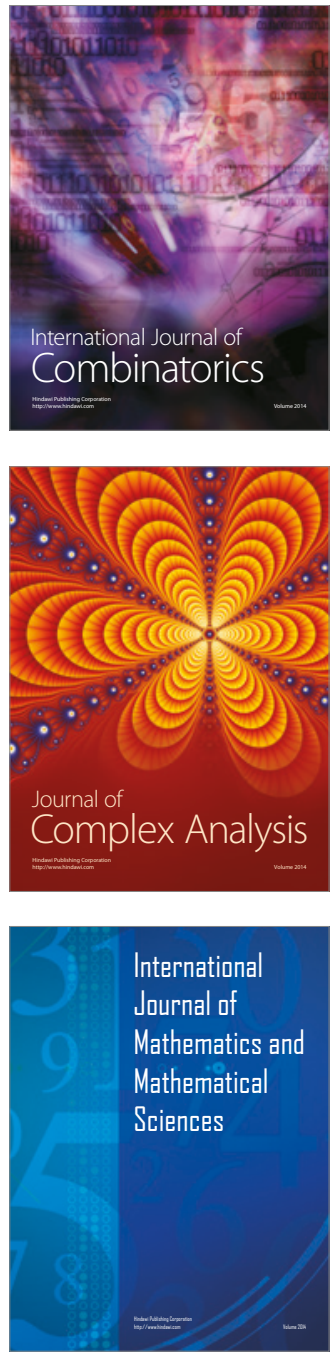
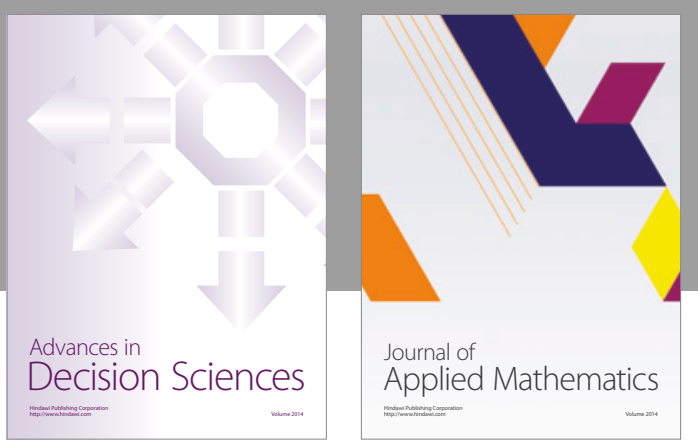

Algebra

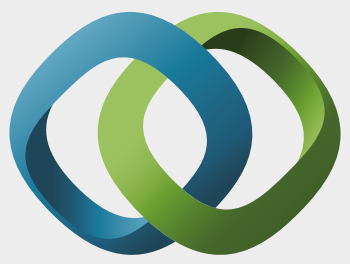

\section{Hindawi}

Submit your manuscripts at

https://www.hindawi.com
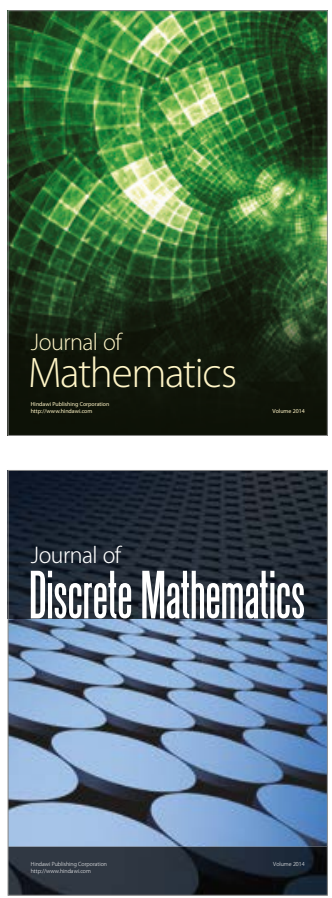

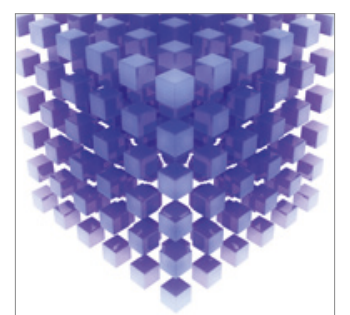

Mathematical Problems in Engineering
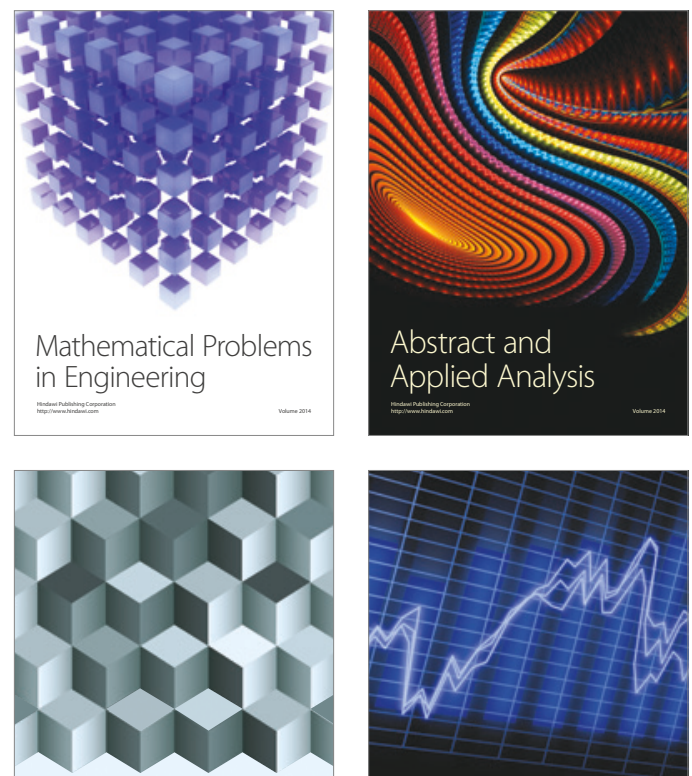

Journal of

Function Spaces

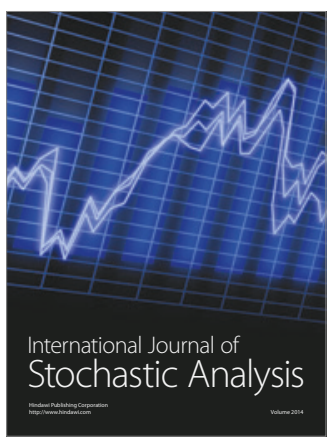

Probability and Statistics
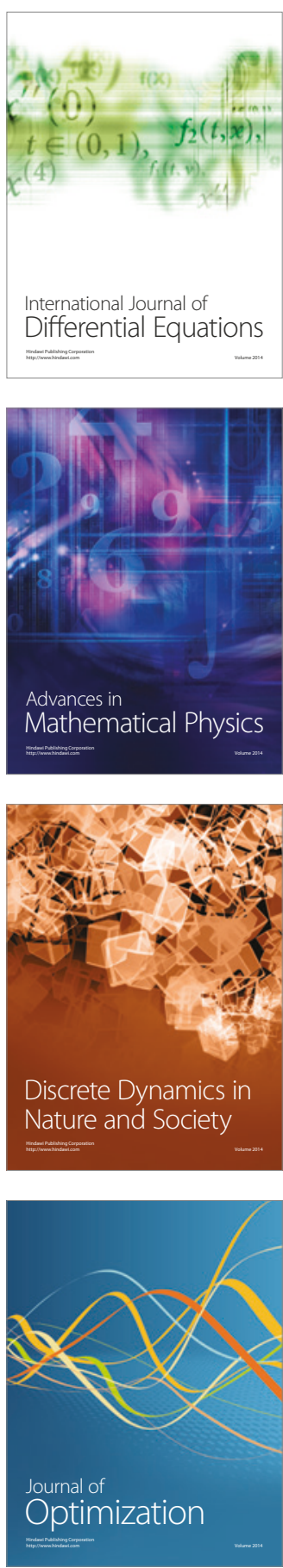